\title{
Three-dimensional analysis of dermal backflow in cancer-related lymphedema using photoacoustic lymphangiography
}

\author{
Anna $\mathrm{Oh}^{1}$, Hiroki Kajita ${ }^{1}$, Nobuaki Imanishi ${ }^{2}$, Hisashi Sakuma ${ }^{3}$, Yoshifumi Takatsume ${ }^{2}$, \\ Keisuke Okabe ${ }^{1}$, Sadakazu Aiso ${ }^{2}$, Kazuo Kishi ${ }^{1}$ \\ Departments of ${ }^{1}$ Plastic and Reconstructive Surgery and ${ }^{2}$ Anatomy, Keio University School of Medicine, Tokyo; ${ }^{3}$ Department of Plastic and \\ Reconstructive Surgery, Tokyo Dental College Ichikawa General Hospital, Ichikawa, Japan
}

\begin{abstract}
Background Dermal backflow (DBF), which refers to lymphatic reflux due to lymphatic valve insufficiency, is a diagnostic finding in lymphedema. However, the three-dimensional structure of DBF remains unknown. Photoacoustic lymphangiography (PAL) is a new technique that enables the visualization of the distribution of light-absorbing molecules, such as hemoglobin or indocyanine green (ICG), and can provide three-dimensional images of superficial lymphatic vessels and the venous system. This study reports the use of PAL to visualize DBF structures in the extremities of patients with lymphedema after cancer surgery.

Methods Patients with a clinical or lymphographic diagnosis of lymphedema who previously underwent surgery for cancer at one of two participating hospitals were included in this study. PAL was performed using the PAI-05 system. ICG was administered subcutaneously in the affected hand or foot, and ICG fluorescence lymphography was performed using a nearinfrared camera system prior to PAL.
\end{abstract}

Results Between April 2018 and January 2019, 21 patients were enrolled and examined using PAL. The DBF was composed of dense, interconnecting, three-dimensional lymphatic vessels. It was classified into three patterns according to the composition of the lymphatic vessels: a linear structure of lymphatic collectors (pattern 1), a network of lymphatic capillaries and lymphatic collectors in an underlying layer (pattern 2), and lymphatic capillaries and precollectors with no lymphatic collectors (pattern 3 ).

Conclusions PAL showed the structure of DBF more precisely than ICG fluorescence lymphography. The use of PAL to visualize DBF assists in understanding the pathophysiology and assessing the severity of cancer-related lymphedema.

Keywords Lymphedema / Lymphography / Superficial lymphatic system

\author{
Correspondence: Anna Oh \\ Department of Plastic and \\ Reconstructive Surgery, Keio \\ University School of Medicine, \\ 35 Shinanomachi, Shinjuku, Tokyo \\ 160-8582, Japan \\ Tel: +81-3-3353-1211 \\ Fax: +81-3-3352-1054 \\ E-mail: prsanna1006@gmail.com
}

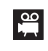

This research was funded by the ImPACT Program of Council for Science, Technology and Innovation (Cabinet Office, Government of Japan).

Received: July 2, $2021 \bullet$ Revised: September 9, $2021 \bullet$ Accepted: September 30, 2021

pISSN: 2234-6163 • elSSN: 2234-6171 • https://doi.org/10.5999/aps.2021.01235• Arch Plast Surg 2022;49:99-107

\section{INTRODUCTION}

Dermal backflow (DBF) is a diagnostic finding of lymphedema that can be observed using indocyanine green (ICG) fluorescence lymphography and lymphoscintigraphy [1-3]. The presence of DBF suggests lymphatic reflux caused by lymphatic

Copyright $\odot 2022$ The Korean Society of Plastic and Reconstructive Surgeons

This is an Open Access article distributed under the terms of the Creative Commons Attribution Non-Commercial License (https://creativecommons.org/

licenses/by-nc/4.0/) which permits unrestricted non-commercial use, distribution, and reproduction in any medium, provided the original work is properly cited. I www.e-aps.org 
valve insufficiency, which develops because of anatomical or functional obstruction of lymphatic pathways $[4,5]$. Surgical treatment for cancer is a common cause of lymphedema, as the structure of the lymphatic system is destroyed during lymph node dissection. Obstruction of lymphatic pathways also occurs after cancer surgery in which components of the lymphatic system are disrupted [4-6].

DBF is believed to be a pathophysiological response that creates alternative pathways for lymphatic flow when the lymphatic pathway is obstructed in patients with lymphedema $[7,8]$. In patients with mild lymphedema, DBF areas are localized as some lymphatic collectors remain functional. In patients with severe lymphedema, most lymphatic collectors are not functional, and the DBF areas increase. Therefore, the pattern and distribution of DBF areas are correlated with the severity of lymphedema $[7,9,10]$.

In terms of the anatomical structure of the lymphatic system, DBF is considered to be composed of lymphatic capillaries in the dermis and precollectors in the deep layer of the dermis [9]. However, since conventional imaging modalities such as ICG fluorescence lymphography or lymphoscintigraphy provide two-dimensional images, DBF is evaluated and classified based on superficial distributions or patterns $[11,12]$. The three-dimensional structure of DBF in patients with lymphedema remains unknown, making it difficult to accurately describe the various findings of DBF.

Photoacoustic lymphangiography (PAL) is a new technique that enables visualization of the distribution of light-absorbing molecules and generates three-dimensional images of superficial lymphatic vessels and the venous system [13]. PAL is based on a photoacoustic imaging technique, in which pulsed laser light is delivered into a light-absorbing tissue component. The delivered light energy results in a transient expansion, generating ultrasonic waves that are detected by the sensor. The signals are then processed to produce three-dimensional images [14].

Photoacoustic imaging has high spatial resolution and the ability to image both endogenous agents (such as oxyhemoglobin, deoxyhemoglobin, and melanin) and exogenous agents (such as ICG). Previous studies [13,15-17] obtained three-dimensional images of superficial lymphatic vessels in healthy participants and lymphedema patients. PAL revealed high spatial resolution images that could identify lymphatic vessels up to $0.2 \mathrm{~mm}$ in size. In addition, DBF could be observed three-dimensionally via PAL images in lymphedema patients [13].

In this study, PAL was used to visualize the three-dimensional structure of DBF and analyze the patterns of DBF in patients with cancer-related lymphedema. This study aimed to investigate the structure and clinical significance of DBF in patients with lymphedema after cancer surgery using PAL.

\section{METHODS}

\section{Patients}

Twenty-one patients with clinically diagnosed extremity lymphedema secondary to cancer surgery were enrolled in this study from April 2018 to January 2019. Two patients who did not have findings of DBF on ICG lymphography were excluded. Therefore, data from 19 patients were analyzed. The duration of lymphedema was calculated from the time that subjective symptoms of lymphedema appeared until enrollment in the study. Clinical staging was conducted based on the criteria in the consensus document of the International Society of Lymphology 2013. Swelling that subsided with limb elevation alone was classified as stage I. Swelling with pitting edema that rarely subsided due to limb elevation was classified as stage II. Stage III involved lymphostatic elephantiasis [18].

This study was approved by the ethics committee of the appropriate ethics review board and was conducted according to the principles of the Declaration of Helsinki of 1975, as revised in 1983. All patients provided written informed consent prior to enrollment.

\section{Imaging device}

A photoacoustic imaging system (PAI-05) was used for PAL in this study. The PAI-05 system is composed of the following equipment: a bed unit including a semi-spherical detector array and a scanning stage, a light source, a fiber bundle, a data acquisition system, a real-time image reconstruction unit, and a personal computer for operation [14]. A semi-spherical detector array detects the photoacoustic signal, which is converted to digital data. The digital data are transferred to a computer inside the PAI-05 for image reconstruction [14]. During PAL examination, real-time images are obtained and displayed on the computer [14]. The maximum measurement range of area is 270 $\mathrm{mm} \times 180 \mathrm{~mm}$ in still images, with a high spatial resolution of $200 \mu \mathrm{m}$. The depth range is up to $20 \mathrm{~mm}$. Thus, a PAL image is generated by a computer in a similar way to a computed tomography scan, and the quality of the obtained image is not operator-dependent. The device we used does not require any special qualifications to perform examinations. However, it is preferable for a specialist such as an operating surgeon to participate in the examination (e.g., if the examination is performed for preoperative planning).

\section{Procedures}

All patients underwent PAL using the PAI-05 system with a 
semi-spherical detector array [13]. PAL was performed by two plastic surgeons. Wavelengths of $797 \mathrm{~nm}$ and $835 \mathrm{~nm}$ were used to image the hemoglobin and ICG distribution. ICG (5 mg/ $\mathrm{mL}, 0.1 \mathrm{~mL}$ of ICG at each site) was administered subcutaneously at three sites on the dorsal aspect of the affected hand or foot. ICG fluorescence lymphography was performed using a near-infrared camera system prior to PAL. The area in which DBF was observed with ICG fluorescence lymphography was marked, and the same area was examined via PAL. PAL images were obtained from two to three areas of the extremity for each patient. The three-dimensional PAL images of DBF were analyzed and compared to the fluorescence lymphography images.

\section{Image interpretation and data analysis}

We used a PAI-dedicated viewer (KURUMI; Kyoto University, Kyoto, Japan) [19] to display the photoacoustic images. We analyzed the PAL images three-dimensionally for the location, distribution pattern, and appearance of DBF, including the relationship with lymphatic collectors. We classified the PAL images into three patterns according to the composition of DBF. Analysis of variance was used to evaluate the differences in characteristics between patients with each pattern of DBF. All statistical calculations were performed using IBM SPSS Statistics version 26 (IBM Corp., Armonk, NY, USA). The threshold for statistical significance was set at $\mathrm{P}<0.05$.

\section{RESULTS}

The mean patient age was $62.4 \pm 13.3$ years (range, $30-81$ years). The mean duration of lymphedema was $9.1 \pm 7.4$ years (range, 4 months-27 years). The mean period from cancer sur-

Table 1. Patient characteristics

\begin{tabular}{lc}
\hline Characteristics & No. (\%) \\
\hline Sex & \\
Male & $4(21)$ \\
Female & $15(79)$ \\
Type of malignancy & \\
Cervical cancer & $6(31)$ \\
Endometrial cancer & $6(31)$ \\
Ovarian cancer & $1(5)$ \\
Breast cancer & $2(10)$ \\
Malignant lymphoma & $2(10)$ \\
Rectal cancer & $1(5)$ \\
Anal cancer & $1(5)$ \\
Adjuvant therapy & \\
None & $11(58)$ \\
Chemotherapy & $7(37)$ \\
Radiotherapy & $1(5)$ \\
History of lymphaticovenular anastomosis & $4(21)$ \\
History of cellulitis & $4(21)$ \\
\hline
\end{tabular}

gery to the PAL examination was $11.94 \pm 9.1$ years (range, $1-31$ years). The patient characteristics are summarized in Table 1. Of the 19 patients, three had unilateral upper extremity lymphedema and 16 had lower extremity lymphedema ( 7 bilateral and 9 unilateral). Three patients were classified as having stage I lymphedema, 14 as stage II, and 2 as stage III.

Nineteen patients were found to have DBF on ICG fluorescence lymphography. The pattern of DBF was identified as an irregularly-shaped high-intensity region on the surface of the affected extremities. According to the classification of DBF in ICG lymphography [11], a splash pattern representing tortuous lymphatic vessels was observed in one patient. A stardust pattern described as a spotted appearance was observed in one patient. In 17 patients, a diffuse pattern was observed, in which the ICG was distributed widely without recognizable lymphatic vessels or spotting (Figs. 1A, 2A, 3A, 4A).

Using PAL, the DBF was visualized three-dimensionally and the findings were classified into three distinct patterns according to the composition of the lymphatic vessels. Five patients showed lymphatic collectors, but not lymphatic capillaries or precollectors, at the site of DBF on the ICG fluorescence lymphography images (Fig. 1B). These patients were classified as pattern 1 (the lymphatic collector pattern).

In this study, 13 patients were found to have DBF using PAL. Each lymphatic vessel that was part of the DBF was clearly observed on the PAL images. DBF was identified as a complex in-



Fig. 1. Lymphedema of the right leg after sigmoid colon cancer surgery. A 73-year-old man presented with lymphedema of the right leg after surgery for sigmoid colon cancer. An indocyanine green fluorescence lymphography image of the medial side of the right lower leg shows dermal backflow (A). A photoacoustic lymphangiography image of the lymphatic collectors is shown (B). 

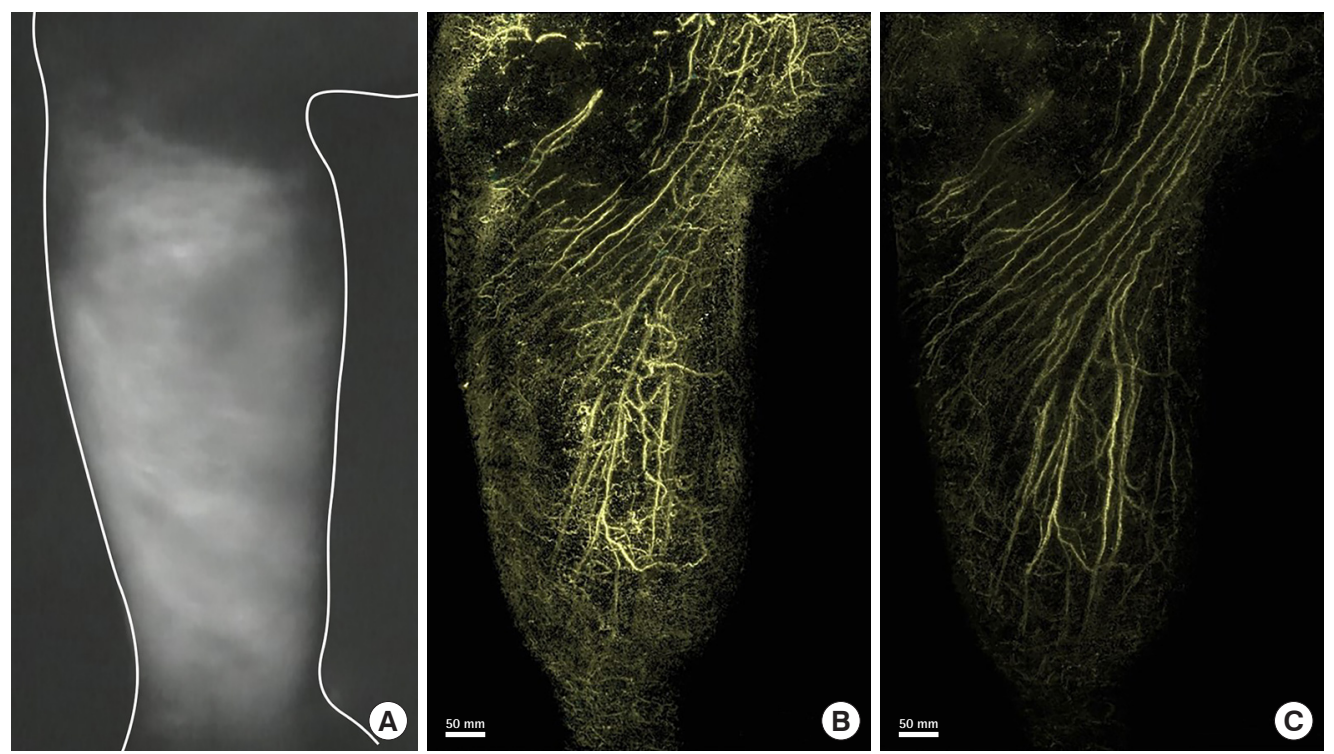

Fig. 2. Lymphedema of the right leg after surgery for lymphoma. An 81-year-old man presented with lymphedema of the right leg after surgery, including pelvic lymph node dissection for lymphoma. An image of the medial side of the right lower leg examined using indocyanine green fluorescence lymphography shows dermal backflow in the entire lower leg (A). A photoacoustic lymphangiography image shows the lymphatic capillaries of the network structure in the superficial layer and a linear appearance of the lymphatic collectors of the deep layer (B). A photoacoustic lymphangiography image of the deep layer shows the lymphatic collectors (C).
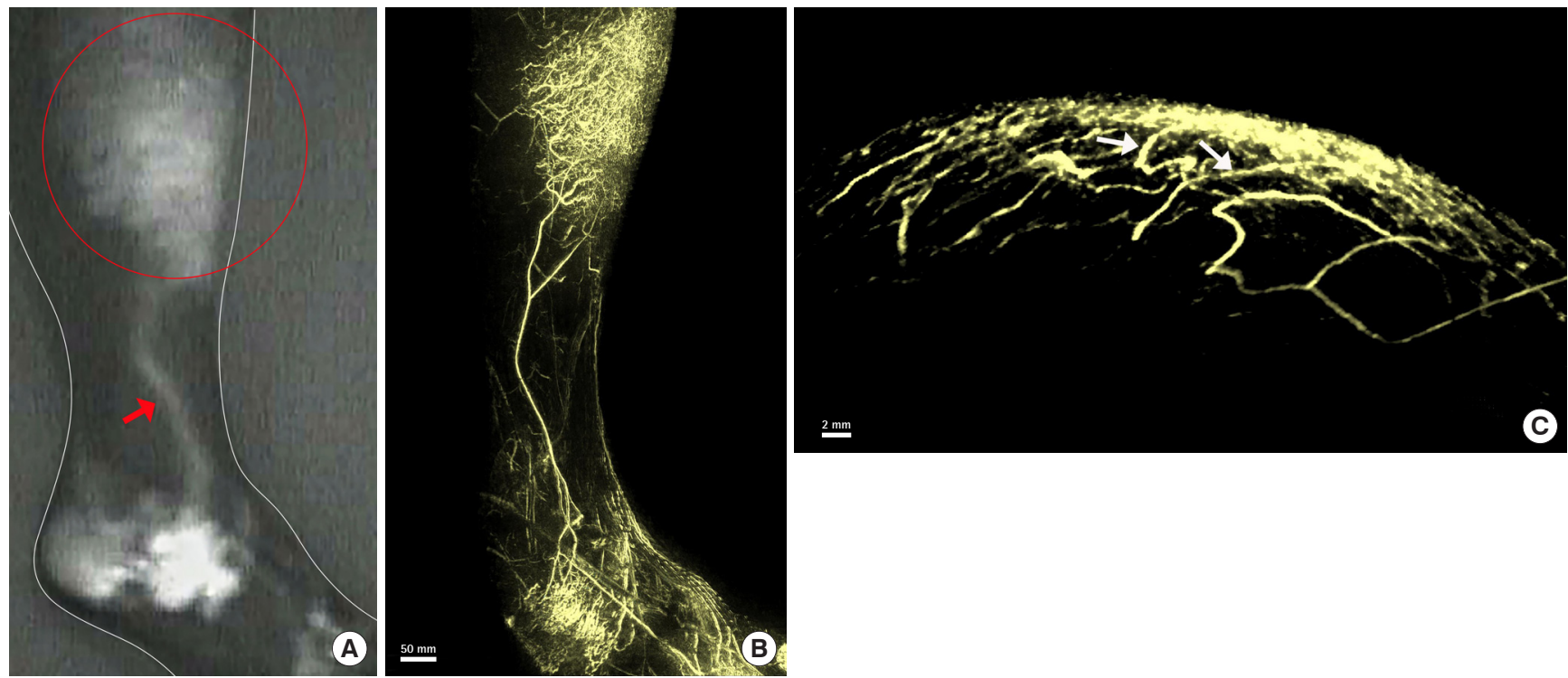

Fig. 3. Lymphedema of the right leg after surgery for cervical cancer. A 30-year-old woman presented with lymphedema of the right leg after surgery for cervical cancer. Indocyanine green fluorescence lymphography images (A) show dermal backflow (DBF) in the proximal right lower leg (red circle), with a linear pattern in the distal area of backflow (red arrow). A photoacoustic lymphangiography image shows the lymphatic capillaries of the network structure and the lymphatic collectors with a linear appearance (B). The lymphatic collectors of the lower leg appear to be connected to the DBF (Supplemental Video 1). An axial-plane image also shows connections (white arrows) between the lymphatic collectors and the DBF (C).

terconnecting structure of lymphatic vessels on the PAL images. Based on the composition of the DBF, two three-dimensional patterns of DBF structure were identified in PAL in these 13 patients. In 10 patients classified as pattern 2, lymphatic vessels were found in the superficial layer, and lymphatic vessels of the deep layer that could not be identified on ICG fluorescence lymphography were observed (Figs. 2B and C, 3B). The structure of the lymphatic vessels in the DBF differed between the superficial and deep layers. The superficial layer of the DBF consisted of lymphatic vessels forming a network structure that 

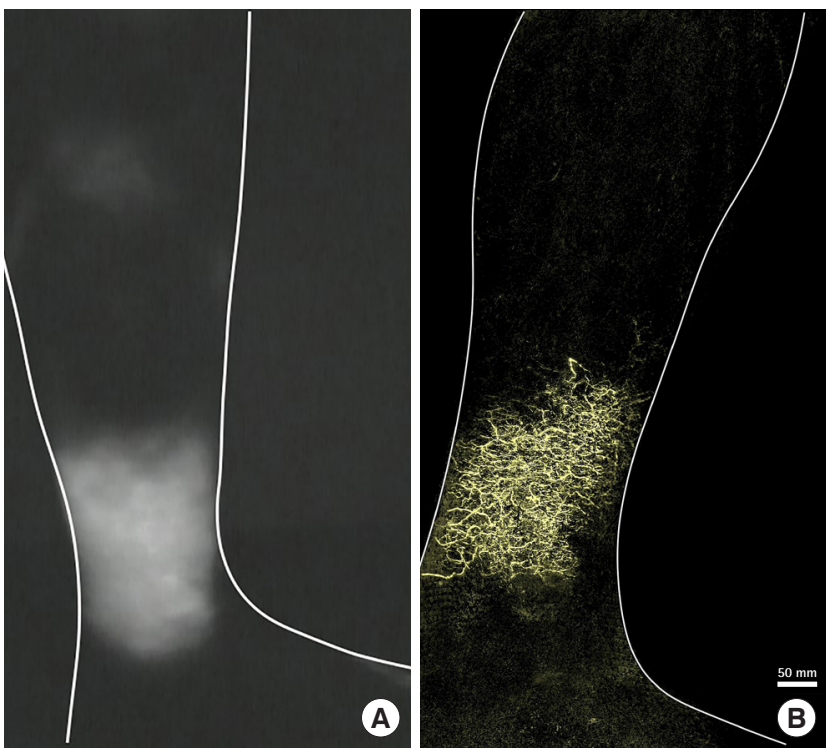

Fig. 4. Lymphedema of the left leg after surgery for ovarian cancer. A 50-year-old woman presented with lymphedema of the left leg after surgery for ovarian cancer. An indocyanine green fluorescence lymphography image of the medial left lower leg shows dermal backflow (A). A photoacoustic lymphangiography image shows the network of lymphatic capillaries without lymphatic collectors (B). was densely intertwined and connected to the lymphatic vessels of the deep layer. The lymphatic vessels that connected the lymphatic vessels in the superficial and deep layers ran perpendicular to the lymphatic vessel axis of the deep layer. The deep layer of the DBF had lymphatic vessels arranged in a linear structure that did not form a network. Lymphatic vessels in this layer ran parallel to each other, joining and branching off at times. Some lymphatic vessels in the deep layer were connected to the lymphatic network in the superficial layer (Fig. 3B and C, Supplemental Video 1), and others were not connected to the superficial lymphatic network. We analyzed the three-dimensional structure of DBF in pattern 2 by their depth (Fig. 5A). The PAL images of each layer coincided with the structure of the lymphatic vessels in the superficial lymphatic system: capillary lymphatics in the dermis, precollectors in the deep layer of the dermis, and lymphatic collectors in the subcutaneous tissue (Fig. $5 B, C$, and D).

In three patients classified as pattern 3 , in which no functional lymphatic collectors remained, only the lymphatic capillaries of the superficial layer were observed without lymphatic collectors of the deep layer (Fig. 4B). In the one remaining patient with
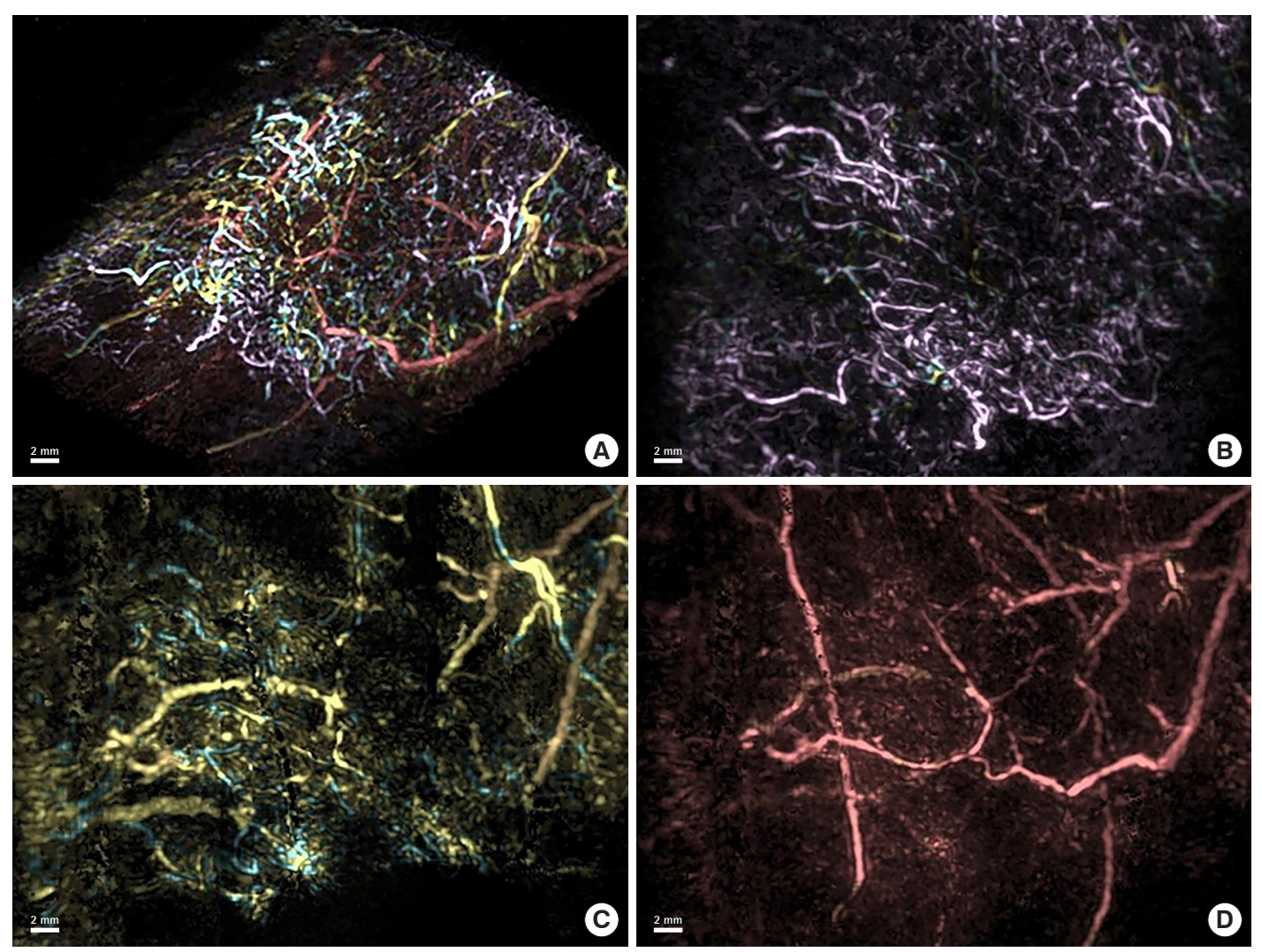

Fig. 5. Three-dimensional image of dermal backflow. A superimposed image on a tilted plane of the three-dimensional structure of dermal backflow is shown with the superficial vessels in pink, the intermediate layer vessels in yellow, and the deep vessels in red (A). Each layer in the sagittal plane is shown individually $(B, C, D)$. Capillary lymphatics are shown in the dermis (pink; B), precollectors are shown in the deep layer of the dermis (yellow; $\mathrm{C}$ ), and lymphatic collectors are shown in the subcutaneous tissue (red; D). 
Table 2. Mean duration of lymphedema and period from surgery to photoacoustic lymphangiography (PAL)

\begin{tabular}{lccc}
\hline Variable & Pattern 1 & Pattern 2 & Pattern 3 \\
\hline No. & 5 & 10 & 3 \\
Duration of lymphedema (yr), mean \pm SD & $4.06 \pm 3.45$ & $8.40 \pm 6.20$ & $20.00 \pm 7.00$ \\
Period from surgery to PAL (yr), mean \pm SD & $5.80 \pm 2.39$ & $12.00 \pm 9.45$ & $22.33 \pm 9.02$ \\
\hline
\end{tabular}

upper extremity lymphedema after breast cancer surgery, ICG lymphography showed a diffuse pattern from the affected hand to the forearm overall. On PAL, some lymphatic collectors were observed in the affected hand, but no lymphatic collectors were observed from the wrist through the entire forearm. This patient was excluded from this classification because few lymphatic collectors were observed in the distal part of the DBF, while patients classified as pattern 1 showed lymphatic collectors in most ranges with DBF.

In this study, PAL was performed on the same region where DBF was observed on ICG fluorescence lymphography. Patients who showed only lymphatic collectors in all obtained PAL images were classified as pattern 1 . In addition, patients who showed features of both pattern 2 and pattern 3 in the examined extremity were ultimately classified as pattern 2 since the remaining function of the lymphatic collector was the defining criterion for classification as pattern 2 or pattern 3. Pattern 3 was restricted to patients in which no functional lymphatic collectors were observed in any PAL images.

The mean duration of lymphedema and the mean period from surgery to PAL are summarized in Table 2 . There was a significant difference in the mean duration of lymphedema among the three patterns $(\mathrm{F}=7.443, \mathrm{P}=0.006)$. Patients with pattern 3 had a significantly longer mean duration of lymphedema than those with pattern $1(\mathrm{P}=0.002)$ or pattern $2(\mathrm{P}=0.008)$. In addition, significant differences were found in the mean period from surgery to PAL between patients with the three patterns of DBF $(\mathrm{F}=3.885, \mathrm{P}=0.044)$.

\section{DISCUSSION}

This study analyzed the three-dimensional structure of DBF and confirmed that DBF is the result of lymphatic backflow from the lymphatic collectors to the precollectors and lymphatic capillaries. PAL enables visualization of lymphatic reflux in the superficial lymphatic system.

In this study, three-dimensional images of DBF were classified into three patterns: lymphatic collectors, a network of lymphatic capillaries and lymphatic collectors, and a network of lymphatic capillaries and precollectors (with no lymphatic collectors). In patients classified as pattern 1 , individual lymphatic collectors were difficult to identify on ICG fluorescence lymphography, as the borders of the lymphatic collectors were blurry. In particular, if multiple lymphatic collectors were located nearby, or if the running pathways crossed each other, the pattern may have appeared diffuse. In these cases, lymphatic vessels such as those shown in pattern 1 run in the range where DBF appears on ICG fluorescence lymphography. These results suggest the possibility that lymphatic reflux into the precollectors or lymphatic capillaries might not actually be present, although the ICG fluorescence lymphography findings are suggestive of DBF. However, it is difficult to conclude that DBF has not occurred only because precollectors and lymphatic capillaries are not observed on PAL. In one patient, who was excluded from our PAL classification, lymphatic vessels were not observed using PAL at all in the part where DBF was shown on ICG lymphography. When lymphatic fluid is refluxed in lymphedema, it finally leaks from the open ends of lymphatic capillaries into the interstitial space in the skin. Compared to ICG fluorescence lymphography, PAL has less sensitivity in terms of detection when the ICG concentration is low. We suggest that PAL was unable to detect ICG spreading into the interstitial space, rather than ICG not being detected because of the absence of lymphatic vessels, since the ICG was diluted. Therefore, we cannot completely exclude the possibility of accompanying lymphatic fluid leakage in patients with pattern 1.

After lymph node dissection, disruption of the lymphatic system impairs lymphatic fluid drainage, causing excess fluid accumulation in the interstitial tissues. The lymphatic system reacts to fluid overload by rerouting lymphatic fluid via DBF and collateral lymphatic collectors. Animal studies have revealed that lymphatic collectors can regenerate to develop the lymphatic pathway [20-22]. However, it is not clear whether the lymphatic collectors observed in PAL were pre-existing or newly developed as collateral lymphatic collectors.

The patterns of DBF networks observed in this study display the conditions in which lymphatic reflux occurs. DBF regulates lymphatic transport via two mechanisms in patients with lymphedema. When functioning lymphatic collectors remain in the affected extremity, as in pattern 2, these structures carry lymphatic fluid between the obstructed and patent lymphatic collectors. When all the lymphatic collectors are obstructed in an affected extremity (pattern 3), DBF serves as an alternative pathway to maintain lymphatic drainage [7]. When lymphatic 
collectors are present, they are found in the deep layer of the DBF. While some lymphatic collectors appear to be connected to the superficial DBF, others are not. Lymphatic collectors that are connected to the DBF may have valve insufficiency in the distal structures of the DBF. PAL images show which lymphatic vessels connect to the DBF, allowing physicians to determine which lymphatic pathway is deteriorated, leading to DBF (Fig. 3C, Supplemental Video 1). This information may improve therapeutic effects, as specific lymphatic collectors can be targeted via lymphaticovenular anastomosis (LVA). In contrast, when lymphatic collectors are not present, the range of DBF is wider, and LVA is not expected to result in symptom improvement. Therefore, lymph node transplantation may be necessary.

ICG fluorescence lymphography is widely used in the diagnosis and evaluation of lymphedema patients. It can show the entire extremity and the function of lymphatic vessels in real-time. However, when evaluating DBF, ICG fluorescence lymphography can only provide the pattern and distribution of the surface in two dimensions. Moreover, it is difficult to specify the relationship with the lymph collectors around the DBF. In addition, due to resolution limitations, each lymphatic vessel cannot be clearly observed. Several previous studies have reported the use of ICG fluorescence lymphography to assess the severity of secondary lymphedema after cancer surgery. These studies suggest that the presence, pattern, and distribution of DBF could serve as a criterion for determining the severity of lymphedema $[11,23]$. In contrast, three-dimensional PAL images enable a more precise evaluation of the severity of lymphedema. The detailed evaluation of DBF provided by PAL makes it possible to create specific treatment plans based on the number of remaining functional lymphatic vessels. The three patterns of lymphatic flow detected using PAL represent the pathophysiology of lymphedema, in which lymphatic flow deteriorates from pattern 1 to pattern 3 . The degree of lymphedema severity is correlated with the amount of functional lymphatic collectors remaining [7]. Notably, these patterns were found to be correlated with the duration of lymphedema and the period from surgery to PAL in this study.

However, PAL has some technical limitations. In PAL, the lymphatic vessels can be observed only up to a depth of $20 \mathrm{~mm}$ from the surface due to the limitation of light penetration [14]; thus, it is difficult to visualize the lymphatic vessels of deep tissues. Furthermore, since the maximum measurement area is also limited, images showing the entire extremity in a single view cannot be obtained. In addition, as shown in the results of this study, it is possible that DBF shown on ICG fluorescence lymphography may not be detected via PAL. Therefore, ICG fluorescence lymphography has better sensitivity than PAL in terms of DBF detection. Meanwhile, the specificity of PAL in DBF detection was difficult to compare with that of ICG in the current results. Therefore, it is necessary to use PAL in combination with conventional devices, such as ICG fluorescence lymphography, in the evaluation of lymphedema to achieve better outcomes.

Ultra-high-frequency ultrasound (UHFUS) $[24,25]$ and magnetic resonance lymphangiography (MRL) $[26,27]$ have been suggested as new techniques for evaluating the lymphatic system. UHFUS can be used intraoperatively in LVA, as it shows lymphatic vessels masked by DBF, which cannot be observed via ICG fluorescence lymphography [25]. However, UHFUS images are limited to a depth of $10 \mathrm{~mm}$ from the surface [24], and UHFUS is not suitable for evaluating DBF. In contrast, MRL can provide an image of the lymphatic vessels in the deep layers, but MRL has a low spatial resolution and it is difficult to distinguish the lymphatic vessels from veins on MRL [26].

It must be emphasized that this was a preliminary study, including a limited number of patients, a limitation that could be addressed in future studies. The current results might not be applicable to determining lymphedema staging, as several factors other than the duration of morbidity contribute to the severity of lymphedema. Further studies with larger numbers of patients are warranted to confirm our results. Nevertheless, our study results were significant in that the DBF shown in secondary lymphedema has a variety of structures that have not been reported in other studies and can be classified into certain patterns.

In conclusion, the use of PAL allows visualization of the threedimensional structures of the lymphatic networks in cancer-related lymphedema. The ability to clinically evaluate lymphatic function after cancer treatment will enable more effective treatment strategies for secondary lymphedema by making it possible to evaluate specific anatomical and functional characteristics of a patient's lymphatic system.

\section{NOTES}

\section{Conflict of interest}

Keio University School of Medicine received grants from the ImPACT Program of Council for Science, Technology and Innovation (Cabinet Office, Government of Japan) and the PAI05 system for this study was provided by Canon, Inc. Keisuke Okabe, Hisashi Sakuma, and Yoshifumi Takatsume have no potential conflicts of interest relevant to this article.

\section{Ethical approval}

The study was approved by the Institutional Review Board of 
Keio University Hospital (IRB No. CRB3180017) and performed in accordance with the principles of the Declaration of Helsinki. Written informed consent was obtained.

\section{Author contribution}

Design and conceptualization of the study: A Oh, H Kajita. Study conduction: A Oh, H Kajita, N Imanishi. Funding acquisition: H Kajita, S Aiso, K Kishi. Recruit patients and clinical databases: A Oh, H Kajita, H Sakuma, K Okabe. Formal analysis: H Kajita, N Imanishi, H Sakuma. imaging data: Y Takatsume, H Kajita. Writing - original draft: A Oh. Writing - review \& editing: H Kajita, N Imanishi, H Sakuma.

\section{ORCID}

Anna Oh

Hiroki Kajita

Nobuaki Imanishi

Hisashi Sakuma

Yoshifumi Takatsume https://orcid.org/0000-0002-9467-2021

Keisuke Okabe

Sadakazu Aiso

Kazuo Kishi

https://orcid.org/0000-0003-1422-2205 https://orcid.org/0000-0002-3267-5884 https://orcid.org/0000-0002-8816-6309 https://orcid.org/0000-0002-6073-6832 https://orcid.org/0000-0001-8614-5195 https://orcid.org/0000-0002-4298-9828 https://orcid.org/0000-0001-8867-5237

\section{Supplementary material}

Supplemental Video 1. Three-dimensional image of right leg lymphedema after surgery for cervical cancer (Fig. 3). The lymphatic collectors from the distal side of the leg appear to be connected to the dermal backflow.

\section{REFERENCES}

1. Rasmussen JC, Tan IC, Marshall MV, et al. Lymphatic imaging in humans with near-infrared fluorescence. Curr Opin Biotechnol 2009;20:74-82.

2. Ter SE, Alavi A, Kim CK, et al. Lymphoscintigraphy: a reliable test for the diagnosis of lymphedema. Clin Nucl Med 1993; 18:646-54.

3. Dylke ES, McEntee MF, Schembri GP, et al. Reliability of a radiological grading system for dermal backflow in lymphoscintigraphy imaging. Acad Radiol 2013;20:758-63.

4. Grada AA, Phillips TJ. Lymphedema: pathophysiology and clinical manifestations. J Am Acad Dermatol 2017;77:100920.

5. Rockson SG. Lymphedema. Am J Med 2001;110:288-95.

6. Aldrich MB, Rasmussen JC, Fife CE, et al. The development and treatment of lymphatic dysfunction in cancer patients and survivors. Cancers (Basel) 2020;12:2280.
7. Suami H. Anatomical theories of the pathophysiology of cancer-related lymphoedema. Cancers (Basel) 2020;12:1338.

8. Chang DW, Suami H. Discussion: comparison of indocyanine green lymphographic findings with the conditions of collecting lymphatic vessels of limbs in patients with lymphedema. Plast Reconstr Surg 2013;132:1619-21.

9. Karacavus S, Yilmaz YK, Ekim H. Clinical significance of lymphoscintigraphy findings in the evaluation of lower extremity lymphedema. Mol Imaging Radionucl Ther 2015; 24:80-4.

10. Suami H, Scaglioni MF. Anatomy of the lymphatic system and the lymphosome concept with reference to lymphedema. Semin Plast Surg 2018;32:5-11.

11. Yamamoto T, Narushima M, Doi K, et al. Characteristic indocyanine green lymphography findings in lower extremity lymphedema: the generation of a novel lymphedema severity staging system using dermal backflow patterns. Plast Reconstr Surg 2011;127:1979-86.

12. Pappalardo M, Lin C, Ho OA, et al. Staging and clinical correlations of lymphoscintigraphy for unilateral gynecological cancer-related lymphedema. J Surg Oncol 2020;121:42234.

13. Kajita H, Oh A, Urano M, et al. Photoacoustic lymphangiography.J Surg Oncol 2020;121:48-50.

14. Nagae K, Asao Y, Sudo Y, et al. Real-time 3D photoacoustic visualization system with a wide field of view for imaging human limbs. F1000Res 2018;7:1813.

15. Oh A, Kajita H, Matoba E, et al. Photoacoustic lymphangiography before and after lymphaticovenular anastomosis. Arch Plast Surg 2021;48:323-8.

16. Suzuki Y, Kajita H, Konishi N, et al. Subcutaneous lymphatic vessels in the lower extremities: comparison between photoacoustic lymphangiography and near-infrared fluorescence lymphangiography. Radiology 2020;295:469-74.

17. Suzuki Y, Kajita H, Oh A, et al. Photoacoustic lymphangiography exhibits advantages over near-infrared fluorescence lymphangiography as a diagnostic tool in patients with lymphedema. J Vasc Surg Venous Lymphat Disord 2021 Aug 2 [Epub]. https://doi.org/10.1016/j.jvsv.2021.07.012.

18. International Society of Lymphology. The diagnosis and treatment of peripheral lymphedema: 2013 Consensus Document of the International Society of Lymphology. Lymphology 2013;46:1-11.

19. Sekiguchi H, Togashi K. Development of rapid MIP viewer for photoacoustic tomography--KURUMI (Kyoto Univ. Rapid and Universal MIP Imager). IEICE Tech Rep 2017;116: 163-7.

20. Blum KS, Proulx ST, Luciani P, et al. Dynamics of lymphatic 
regeneration and flow patterns after lymph node dissection. Breast Cancer Res Treat 2013;139:81-6.

21. Suami H, Scaglioni MF, Dixon KA, et al. Interaction between vascularized lymph node transfer and recipient lymphatics after lymph node dissection: a pilot study in a canine model. J Surg Res 2016;204:418-27.

22. Tashiro K, Shibata T, Mito D, et al. Indocyanine green lymphographic signs of lymphatic collateral formation in lower extremity lymphedema after cancer resection. Ann Plast Surg 2016;77:213-6.

23. Tashiro K, Yamashita S, Saito T, et al. Proximal and distal patterns: different spreading patterns of indocyanine green lymphography in secondary lower extremity lymphedema. J Plast Reconstr Aesthet Surg 2016;69:368-75.
24. Bianchi A, Visconti G, Hayashi A, et al. Ultra-high frequency ultrasound imaging of lymphatic channels correlates with their histological features: a step forward in lymphatic surgery.J Plast Reconstr Aesthet Surg 2020;73:1622-9.

25. Hayashi A, Visconti G, Yamamoto T, et al. Intraoperative imaging of lymphatic vessel using ultra high-frequency ultrasound. J Plast Reconstr Aesthet Surg 2018;71:778-80.

26. Lu Q, Delproposto Z, Hu A, et al. MR lymphography of lymphatic vessels in lower extremity with gynecologic oncology-related lymphedema. PLoS One 2012;7:e50319.

27. Neligan PC, Kung TA, Maki JH. MR lymphangiography in the treatment of lymphedema. J Surg Oncol 2017;115:1822. 